\title{
Forensic Analysis Algorithm: By using the Tiled Bitmap with Audit Log Mechanism
}

\author{
Piyush P.Gawali \\ Prof.Ram meghe institute of Technology \& Research, Badnera \\ Maharashtra, India
}

\begin{abstract}
The set of policies and the group of people need to access the valuable database by the authorized inter mediator, still the organizational employee also go through the authorized inter mediator. Cryptographic hashing is one of the best approach and work as a inter mediator. This paper show how to resolve when tampering arises in Database, what data was tampered and the identification of the person to tamper the data. These things are detected by using the forensic analysis. This paper presents a new forensic analysis algorithm, the tiled bitmap algorithm, which is more capable then the previous algorithm. It introduces the concept of a candidate set (all possible place of detected tampering(s)) and gives a full classification of the candidate set and its cardinality and prevent the intruder, the computing of the candidate set is also presented.
\end{abstract}

There are certain cases happened in BANK and other sector where the data has been tamper by the assessor, outsider or by the employees of the organizations. The separate audit logs validate to observe and inspect the database along with the extra information and state of the data. Audit log play a central role in database. The space and time complexity is less in this forensic analysis algorithm.

\section{General Terms}

Forensic Analysis Algorithm by Tiled Bitmap with audit Log, Performance, Security, Temporal Database.

\section{Keywords}

Compliant Record, Tiled Bitmap Algorithm, database tampering, Forensic cost, Database Management, integrity and protection, Validator, candidate set, audited table, MD5.

\section{INTRODUCTION}

The suitable current central laws (i.e. federal laws) HIPAAACT [12] (Health Insurance Portability and Accountability), PIPEDA Canada act and the association of widespread news between the assistant and the companies they audit (e.g., Enron, WorldCom) helped to accelerate recent passage of federal laws and official better control on electronic data and The passive record are those necessaries by the countless laws and the policy. The main important point of this paper is to destruct of database protection threat and this threat can rise above during the Database Forensic and there is a enormous quantity of self-directed hazard happen to store the more secret data into the database and there are lots of large organization are failure to inspect the data and data contravene. There are variety of risks create for the database security like Finance control, the nature of threat, improper inter departmental collaboration; lot of IT persons access the core database, limited number of Database security professionals.

Cryptographically strong one-way hash functions agree to the finding of a corruption event (CE), which is several event that violate the data and conciliation of database. Due to enemy as well as auditor or employee or even unfamiliar bug in software or hardware crash corruption event occurs [10].

\section{RELATED WORK}

Widespread news coverage of collusion between auditors and companies they audit [1], a recent FBI study indicates that almost half of attacks were by insiders [2].It is assumed that the notarization and validation services remain in a trusted computing base. This can be done by making them geographically and perhaps organizationally separate from the DBMS and the database [3], thereby effecting correct tamper detection even when the tampering is done by highly motivated insiders. Scenario, like discusses tampering event in which in U.S., all patients are required to sign an authorization under HIPAA [4].Computer forensics is now an active field, with more than 50 books published in the last 10 years. There are few computer tools for these tasks, in part due to the heterogeneity of the data. One substantive example of how computer tools can be used for forensic analysis is Mena's book [5]. Goodrich et al. introduce new techniques for using main-memory indexing structures for data forensics[6].In the database context, previous papers introduced the approach of using cryptographic hash functions to detect database tampering [7] and of introducing additional Hash chains to improve forensic analysis [7]. Previously, there has been proposed the Monochromatic, RGB, and Polychromatic forensic analysis algorithms [8].

If an adversary modifies even single byte of data or its timestamp, the independent Validator will detect mismatch with the notarized document, thereby detecting the tampering. The adversary could simply re-execute transactions, making whatever changes he/she wanted, and then replace original database with his/her altered one. However, the notarized document would not match in time. Avoiding tamper detection comes down to inverting the cryptographically strong one way hash function. An extensive presentation of an approach, performance limitations, tamper detection, threat model and other forensic analysis algorithms is discussed in paper[7],[9].Hash chain linking is discussed in more detail in paper[7].Tiled bitmap algorithm is refinement of polychromatic algorithm. The advantage of the Tiled Bitmap Algorithm is that it lays down a regular pattern (a "tile") of such chains over contiguous segments of the database [14]. The other advantage of the Tiled Bitmap Algorithm is that it can detect multiple corruption events that other previous algorithms can-not. On the other hand it suffers from false 
positives while the previous algorithms do not. There are many models have been proposed to find the tamper detection process like

\subsection{Monochromatic Algorithm}

The Monochromatic Algorithm uses only the cumulative (black) hash chains seen so far, and as such it is the simplest algorithm in terms of implementation.

\subsection{RGB Algorithm}

In the RGB Algorithm, three new types' chains are added, denoted with the colors red, green, and blue, to the original (black) chain in the so-called Monochromatic Algorithm. These hash chains can be computed in parallel; all consist of linked sequences of hash values of individual transactions in commit order. While additional hash values must be computed, no additional disk reads are required. The additional processing is entirely in main memory. The RGBY Algorithm retains the red, green, and blue chains and adds a yellow chain. This renders the new algorithm more regular and more powerful.

\subsection{RGBY Algorithm}

The RGBY Algorithm is an improvement of the original RGB Algorithm. The main insight of the previously presented RedGreen-Blue forensic analysis algorithm (or simply, the RGB Algorithm) is that during notarization events, in addition to reconstructing the entire hash chain (Illustrated with the long right-pointed arrows in prior corruption diagrams), the Validator can also rehash portions of the database and notarize those values, separately from the full chain.

\subsection{A3D Algorithm}

The a3D Algorithm is the most advanced algorithm in the sense that it does not lay repeatedly a "fixed" pattern of hash chains over the database. Instead, the lengths of the partial hash chains change (decrease or increase) as the transaction time increases, in such as way so that at each point in time a complete binary tree (or forest) of hash chains exists on top of the database. This enables forensic analysis to be speed up significantly. In all the above mentioned algorithms they differ in the amount of work necessary during normal processing. Monochromatic algorithm uses an array Black Chains of Boolean values to store the results of validation during forensic analysis. Computing additional hash chains during periodic validation) and the precision of the when and what estimates produced by forensic analysis. The Boolean results are indexed by the subscript of the notarization event considered: the result of validating is stored at a given index. Since do not wish to pre compute all this information, the validation results are Computed lazily, i.e., whenever needed. This can give rise to corruption easily. The RGBY Algorithm was designed so that it attempts to find more than one.

\subsection{Tiled Bitmap Algorithm}

This algorithm introduces the notion of a candidate set (all possible locations of detected tampering(s)) and provides a complete characterization of the candidate set And its cardinality. An optimal algorithm for computing the candidate set is also presented. Finally, the implementation of the Tiled Bitmap Algorithm is discussed, along with comparison to other forensic algorithms in terms of space/time complexity and cost. Where candidate Set Function is to arrange values of targeted binary array in reverse order and renumber function is to re arrange values of targeted binary array imperfect order. So in our proposed System the DBMS computes a cryptographically strong one-way hash function for each tuple inserted and then notarizes it using a notarization service. This made it possible to check the consistency of the data by comparing it to the values stored with the notarization service. In continuation with this method, algorithms were designed to further analyze an intrusion of database [14].

\section{DIFFERENT FORENSIC STEPS:}

Authenticated and Authorized user access the data by using various mechanisms provided by the Database Server. But some time the authorized user makes the data get tampered, so the system is also not secured and protected. Authorized user directly access the Database by using some legal act but authorized user also access the database with the help of IP address and try to make some modification in the database like changes in item price and changes in item quantity and this changes provides the financial loss that's why Database server do not promise for the true data. Due to this issue there is the need of the Forensic Analysis Algorithm. The authorized and unauthorized user detected by the Tiled Bitmap forensic analysis algorithm [14].The Forensic Analysis method is logically planned. During the Digital Analysis of the Database number of operation is executed and Forensic Analysis will take care whether this operation is executed in sequential manner or not. The Forensic Analysis also collects the data during the analysis and operation execution and this data is needed to be submitted as evidence. The following three steps consider during the forensic investigation

STEP 1: Data dictionary is the most important part and the target of the attacker need to make subtle changes in Data Dictionary. Data Dictionary also contains information, such as creation time of entity. The Forensic analysis algorithm uses this information for the investigation.

STEP 2: During the forensic investigation number of users created number of different schemas and these schemas may relevant.

STEP 3: Audit log or Metadata or communication between this is use to find who is the authorized to perform certain action. Data mining tool provide valuable help in Forensic analysis algorithm.

\section{TAMPER DETECTION PROCESS}

With the Database there are several things and ideas come with the database operation. This ideas concert allows Tamper Detection.

The following process was used in tamper detection model to find the database tampering.

The First Process: Audit log maintain by the DBMS itself as a background. This background audit log representing individual relation and this individual relation is treated as a Transaction Time table. In DBMS update, delete and modify operation perform on data (Tuple) if this operation take long time then the Audit log and Transaction time table Drill the DBMS to keep the previous tuple during this operation with their insertion and deletion/update time. During this The DBMS provide one important property with the stored Data in database that it is Modification. If want to modify the only add information at End no information is Deleted. If the old information was changed that time the data get tampered.

The Second Process: The Transaction made the cryptographically hash for the modify data to generate the secure one-way hash of Transaction. 
The Third Process: By using the external notarization service the hash value notarize because of this the intruder, operating system and hardware cannot change the hash value. If the intruder, operating system and hardware makes any Changes in hash value it is very difficult to make the hash value for this change hash value regarding to the Audit Log.

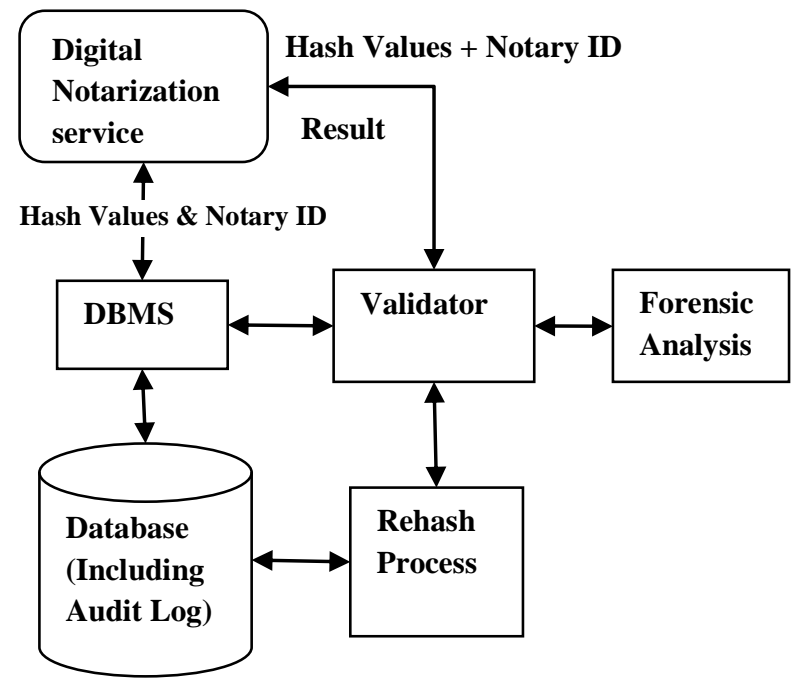

Fig 1: Online Processing with audit log validation

The Fourth Process: Finally the matching is performed between old hash values with rehash tuple. If hash value is same there is no problem but if matching is not occurred then forensic analysis algorithm apply to find out where, when and why the tampering has been occurred.

\section{PROPOSED MODEL}

The proposed model describes how to collect the Data and provide the security of this data. It also provides the basic element about the tampered Data. The audited table separately stored in DW (Data warehouse) and the client signature also stored in Data warehouse.

Let's analyze this model with additional information.

1) A User will officially or unofficially create Tampering. That User Information stored in separate DW (Data warehouse).

2) Validation Component provides Locking Mechanism and the Locking mechanism LOCK the all secured collected Audit Logs.

3) By using the SQL the different operation (INSERT, UPDATE, and DELETE) happen with the database. If modification wants to perform, this modification happens in background of the Database. User plays with this operation and modification by using the certain application, so the user request goes through the application layer and call the SQL to execute the procedure of operations [18].

4) During INSERT operation into Audi table, trigger evaluates two hash values and stores with every record. Figure 3 describes this mechanism in more details [16].

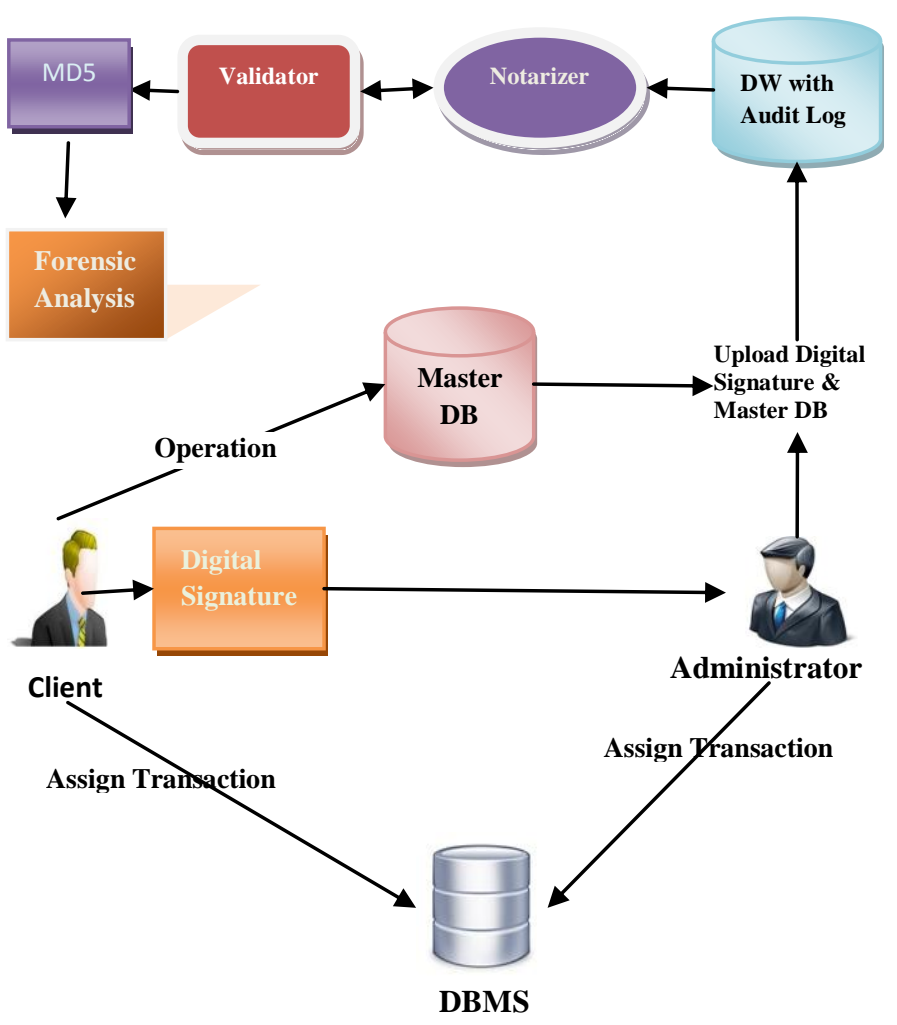

Fig 2: Tamper Detection Model

The submission of request goes to the DATABASE by using the SQL, as discuss above the submission of request goes through the application layer is not the last fragment of Information system or the DBMS After the submission the detection is generated with the SQL prompt. Prompt is the schedule of encoding of program and this prompt assign with the event and the SQL prompt implemented in special SQL code. The SQL prompt executed automatically.DDL prompt is also one important part in RDBMS, some of DDL prompt is specially bunch together and make the group of this special DDL Prompt. 5) In RDBMS the Database objects is created, if someone wants to make any changes in database that time the DDL prompt is executed. There are two special columns called HReserved and VReserved as shown in Figure 3 below. The algorithm involving these two columns are in a way that whenever there is an insert operation in the Audit Log table two hash values - a row hash, and a column hash of this table is calculated. The final Fragment is the security, and each and every record pass through the last fragment the last fragment is created with the cryptographically one way hashing function to provide the hash value for each every individual row. This Fragment is also used for the storing the audit log so when the data was entered into the audit table the cryptographic method and triggers generate the two values, one value for the ROW and another value for COLUMN. The table in database is nothing but the collection of ROWS and COLUMNS. for each and every individual ROW separate column is there and the name of this column is HReserved (HR) and another separate column for each and every individual column and name of this column is VReserved (VR).The row is stored in HR and this HR contain hash value for all column without including HR and VR if Data available in a row was change or modify the data so this modification or change provide new hash value so mismatch Is performed Within old hash value and new hash value and the tampering is detected. The CV contain column hash value, and this hash 
value based on RV.IF the intruder delete a row form the audit table the algorithm find a mismatch by the continuation of new two rows immediately foregoing the deleted row[18].

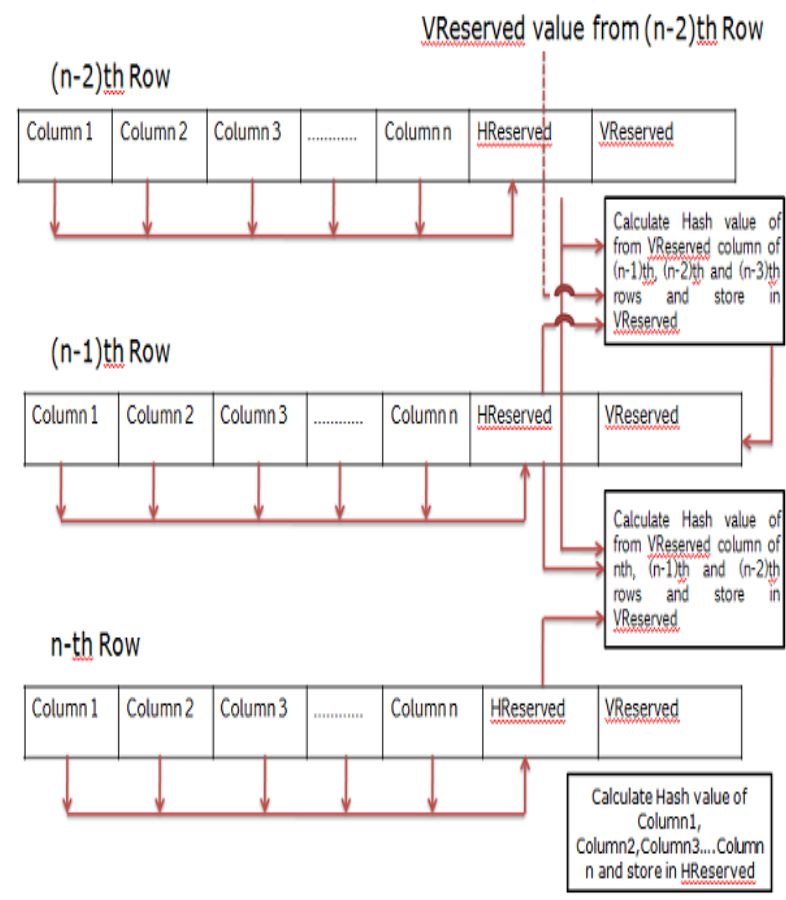

Fig 3: Audit Log Technique

The tiled bitmap algorithm [14] is the modified version of the polychromatic algorithm [15], the group of a sequence value (hash chain) and there is some time gap between these hashes chain and this hash chain coupled with this time gap and real validation interval. Below figure 4 shows the validation happens every 16 hours, during this time thousand even millions of transaction occur, and below figure 4 shows one 16-hour fragment (slice).time proceed from left to right with the hour shown as $r=0$ to $r=15$ and in second fragment hour 17 match up with $r=0$. hour 28 match up with $r=11$ and below figure 4 also demonstrate a corruption event and in this corruption event the timestamp of tuple relation was changed from 31 to 28 hour. consider this relation contain confidential information and when this confidential information discharge authorization were sign by the patient, in this case authorization was sign in hour 31.an hour 29 a doctor expose the patient health information to insurance company and he recognize his mistake, actually this is the crime under act of HIPAA[1].by using the left pointing arrow on hour 28 the authorization backdated and database entail that the authorization received before confidential information convey.

The forensic analysis algorithm hamper the corruption event and also restrict the corruption within the every hour, as shown in figure 4 there are five hash chain is available from $\mathrm{c} 0$ through $\mathrm{c} 4$. The $\mathrm{c} 0$ has chain hashes all transaction with the notarization interval of 16 hour and $\mathrm{c} 1$ hash chain hashes the transaction only for the first 8 hour, $\mathrm{c} 4$ hash chain hashes the other hour transaction. The dotted line indicates the communication of hash chains. as shown in figure 4 in the hash chain $\mathrm{c} 4$ the last transaction hash value of hour 0 hashed with the first transaction of hour 2[5].For the tamper detection after some hour all hash chain are recomputed on tampered data and this recomputed value send to the notarize server, the server find the matching between the old and new hash value
If matching is found there is no tampering in data and unmatched value shows the tampering is occurred.

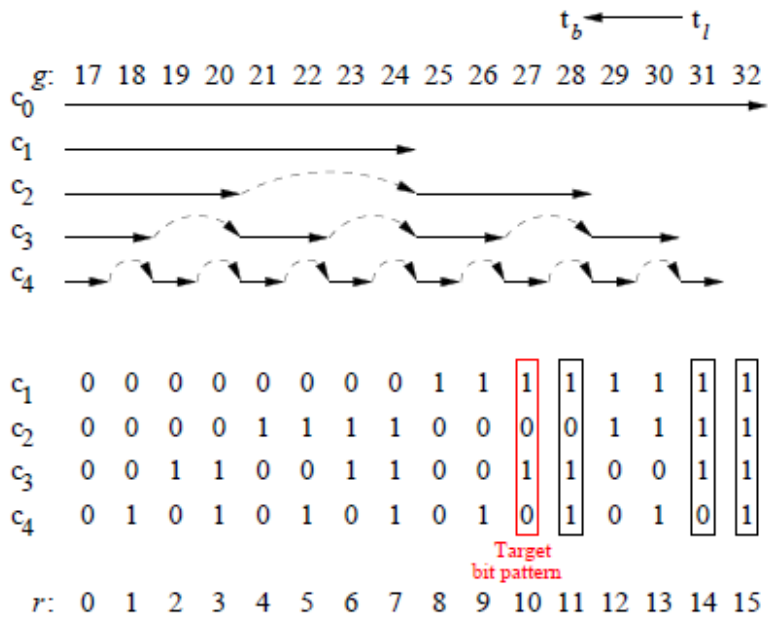

\section{Fig 4: Hash Chain}

By using the forensic analysis algorithm the hash chain c0 report during the 16 hour the tampering is occurred The remaining four chain compute the 4-bit value based on the corruption event and this 4 bit value is 1010 .the $\mathrm{c} 1$ hash chain value and c 3 hash chain value is not affected by the corruption but hash chain value for $\mathrm{c} 2$ and $\mathrm{c} 4$ is not matching with previous value. the truth value as shown in figure 4 the target string would result had the corruption event tampered with the data stored at the indicated hour and also the timestamp changed from tl to tb from 31 to 28 and corruption event occurred at hour 47 .In forensic analysis algorithm the our targeted binary value is 1010.changing the data of 1 hour of interval make all of chain as a failure.

The target 1010 indicates the several possibilities.

Possibility 1: The first possibilities is the only the data in hour 27 was modified $(\mathrm{r}=10)$.

Possibility 2: The second possibilities is the timestamp move from 28 to $31(r=11$ to $r=14)$.

Possibility 3: The third possibility is the timestamp move from 31 to 28 .

Possibility 4: The fourth possibility is the change from hour 27 to 31 , a change from 32 to 27 .

\section{PROPOSED METHOD}

In this section, the described steps show the systematic approach of forensic analysis to find the database tampering.

There are 13 main steps available in our approach.

Step 1, 2, and 3: In This step's the DBA (Database Administrator) give permission to client for the Database Operation, This client's digital signature is created by using the SHA-1 algorithm with the DSA. This signature is in encrypted form.

Step 4, 5 and 6: In these steps upload both the master data on which operations need to be performed \& also a digital signature. When Client want to perform any operation regarding to Database with refer to master database that time the client digital signature is verified. The Client signature and master database data stored in a specific location of the web 
server of DW (data ware house).The Audit Log is also provided with the Data warehouse.

Step 7: As shown in figure 1 The Client and DBA Assign Transaction to the DBMS Then all the data field enter Through the Web application. The separate uploading panel provides to the clients and the DBA and these panels temporally stored in java bean classes.

Step 8: Through the Administrator the digital signature is uploaded in Data warehouse and this is act as notarize element for each and every transactions, whenever the transactions occurs notarize confirm the private key for those Transactions. If private key is same as provided by the Administrator then the transaction completed successfully otherwise the transaction rejected.

Step 9: The Validate conveys the transaction details to notarize, by using these details notarize authorize the data.

Step 10: After the Data confirmation through the Validator, the strong cryptographically one way hashing is performed on this data with the MD5 Algorithm which gives a sixteen byte hash value for both the master data $\&$ also for the transaction data either by the auditor or by the organization employee.

Step 11 and 12: In this step the hash value is check in between the master data and transaction data if value is same there is no tampering is occurred but if the hash value is different in between the master and transaction data then the MD5 algorithm check whether this un matching produce a very large amount effect on dataset in this way to detect data tampering for the several data owner for their own data with their respective applications with their respective signature.

Step 13:In this step the forensic analysis performed on each and every individual tampered tuple to find who tampered the data, what time tampering has been has been occurred and the field where tampering has been happened.

Here in our projected algorithm it admit Master data set and transaction data set after that produce a one more set called Dset which truly consists of array of data field index whose value will be setting to begin with " 0 ". This specifies that the fields are not yet tampered.

Then for each master data set Mset and for each transaction data set Tset our algorithm capture each data fields of these two sets and match up both of them. If they are not equal then that data field is considered as tampered and then di that belongs to Dset is set to value " 1 ". This way the complete tuple is continue checking for the correct tampered fields and tampered person name can be discover using servlet which actually set the user name as he/ she login into the system and by using date and time operation on the same case calculate the accurately at time data tampering is been occur.

Propose the above Discussed Forensic Analysis method by using following Algorithm:

// input: //MDs set is the set of master database

// Mdh Master Database Hash Value

// TDs is the set of Transaction database

// Tdh Transaction Database Hash Value

// Dset is the set of Data Field Index

$/ / \mathrm{Ne}$ Notarize Element

// UN is Username

//Dsign Digital Signature by using SHA-1Treated as a Private Key

// td is date and time
// Rset is the set of Result

// output: Rset the set of Result

Function forensic Analysis (MDs, TDs, Dset, UN, td, Rset)

1: Client Dsign Created (Private Key)

2: For Each transaction

If Dsign is same

\{Assign Transaction\}

Else \{Denies Transaction $\}$

3: $\mathrm{di}=0 / /$ data field Index

4: Initially Result Set Empty Rset="'

5: for $\mathrm{i}=1$ to number of data fields

6: Tdh Transaction Database Hash Value

7: Mdh Master Database Hash Value

8: ifTdh! $=$ Mdh

9: $\mathrm{di}=1$

10: end of for

11: for $\mathrm{i}=1$ to number of data fields

12: if $\mathrm{di}=1$

13: Rset $=$ Rset + di

14:ReturnRset-

\section{ESTIMATION AND METHODOLOGY}

\subsection{Time Complexity}

The Monochromatic Running time complexity is $\mathrm{O}$ $(\log (\mathrm{D} / \mathrm{Iv}))$, RGB Running Time complexity is $\mathrm{O}(\mathrm{D} / \mathrm{Iv})$, Tiled bitmap running time complexity is $\mathrm{O}((\mathrm{D}$.Ig $\mathrm{Iv}) / \mathrm{Iv}+\mathrm{D})$. The Polychromatic algorithm is not here because it is exchanged with the tiled Bitmap Algorithm beside with our approach. With the time our algorithm is gradually slower because of the Chain enlargement and the use of this enlarge chain in our algorithm. As compare to our approach the Monochromatic is fastest one but this algorithm identify only the first corruption event. The Tiled Bitmap Algorithm is somewhat extent in improved manner, this algorithm retain the enlarge chain of Monochromatic algorithm and this algorithm order the corrupted tiles by executing the binary search. This Modification not disturb the asymptotic Running time. Our algorithm is very time consuming because algorithm finds the corruption event on each and every transaction.

So Time Complexity of our approach: O $(\log (\mathrm{D} / \mathrm{Iv}))$.

\subsection{Space Complexity}

The Space complexity of Monochromatic and RGB Algorithm is O (D) but for the suitable validation interval IV the space complexity is cost is higher. In Tiled bitmap algorithm the cost for this validation interval IV is smaller but in our approach the cost for this validation interval IV is smaller as compare to Tiled Bitmap algorithm. This cost also affects the space complexity of forensic analysis algorithm.

Space Complexity of Tiled Bitmap Algorithm (D. (1+lg IV)/IV).

Space Complexity of our approach :( D. (log IV)/IV)

\subsection{Invention Scrutiny}

The Tiled Bitmap Algorithm [14] recommended that the tamper detection complete only for the tiles but in our approach perform the tamper detection on live data. In our approach Master Database is strong and regimented to sustain the structure and also communicate with the all tools available in system. For the Future scope this Database simply expandable. This Algorithm gives a systematic path to the employee and auditor for the secure communication with the system. By using this algorithm stop the database disturbance 
form insider's and the outsider's. Because of the audit $\log$ it is capably auditing the central database.

\section{Result, Comparison and Flowchart}

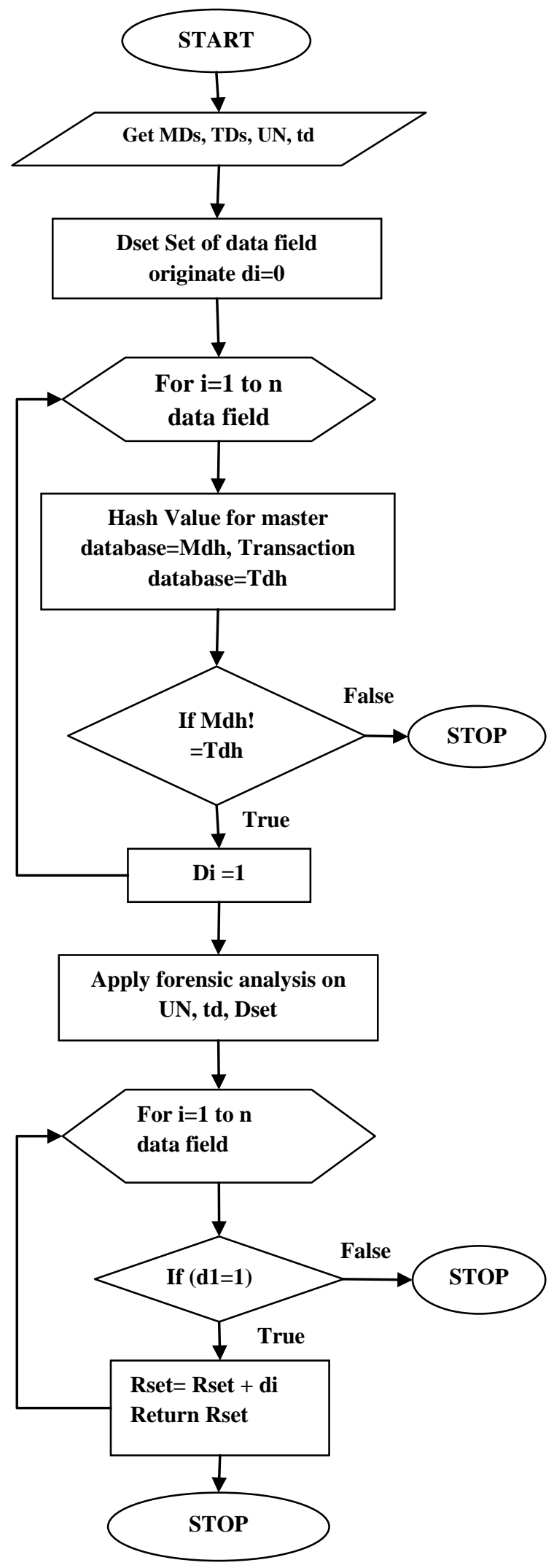

Proposed Algorithm Flowchart
Table 1 Result of our approach

\begin{tabular}{|c|c|c|c|}
\hline Sr.No. & Person Name & Date \& Time & Data Field \\
\hline 1 & Piyush & $\begin{array}{c}15 / 01 / 2013,01: 23 \\
\text { PM }\end{array}$ & Author Name \\
\hline 2 & Shraddha & $\begin{array}{c}16 / 01 / 2013,03: 45 \\
\text { PM }\end{array}$ & Discount \\
\hline 3 & Sais & $\begin{array}{c}17 / 01 / 2013,04: 00 \\
\text { PM }\end{array}$ & Discount \\
\hline
\end{tabular}

Table 2 Comparison

\begin{tabular}{|c|c|c|c|}
\hline Algorithm & Time & Space & Cost \\
\hline RGB & Low & More & High \\
\hline Monochromatic & Fast & More & High \\
\hline A3d & Low & Medium & Medium \\
\hline Tiled Bitmap & Medium & Less & Low \\
\hline Our Approach & Fast & Less & Low \\
\hline
\end{tabular}

\section{CONCLUSION}

The audit log approach is used for the transaction processing method because of this approach the algorithm is capable to detect tampering. Our approach is based on cryptographically one way hashing function, notarization service, and validator. The present paper develops upon that work by present the Tiled Bitmap Algorithm which is cheaper and more commanding than previous algorithms. The notarization service communicates with each and every individual tuple interaction reduces by hashing function and sequence number of tuple is used for better hashing Because of audit log activities the additional data stored in audited table.Validator gives the audited table data because the audited table the validator occasionally relate to the notarization service. If audit $\log$ is tampered the forensic analysis algorithm is activated and going back to checks the other records due to this fashion easily determine who Attack, What Data tampered and why tampered the data.

Thinking from the attacker's point of view this paper gives a contribution towards the identification of the general locations in a database for collecting the digital evidences.

\section{ACKNOWLEDGEMENT}

I am very thankful to the people those who have provided me continuous encouragement and support to all the stages and ideas visualize. I am very much grateful to the entire PRMIT\&R group for giving me all facilities and work environment which enable me to complete my task. I express my sincere thanks to Dr.S.R.Gupta, Dr.G.R.Bamnote Head of computer Department PRMIT\&R Badnera who gave me their valuable and rich guidance and help in presentation of this research paper.

If I forget to give thanks as well as if I forget to mention reference name of anybody in reference list I apologies for that.

\section{REFERENCES}

[1] CSI/FBI, "Tenth Annual Computer Crime and Security Survey,”July2005,http://www.cpppe.umd.edu/Bookstore/ Documents/2005CSISurvey.pdf (accessed April 16, 2009). 
[2] P. A. Gerr, B. Babineau, and P. C. Gordon, "Compliance: the effect on information management and the storage industry, "Enterprise Storage Group Technical Report, May

2003,http://www.enterprisestrategygroup.com/ESGPubli cations/ReportDetail.asp?ReportID=201 (accessed April 21, 2009).

[3] M. T. Goodrich, M. J. Atallah, and R. Tamassia, "Indexing Information for Data Forensics," in Proceedings of the Conference on Applied Cryptography and Network Security, Springer Lecture Notes in Computer Science 3531, pp. 206-221, 2005.

[4] B. Li, M. S. Hsiao, and S. Sheng, "A Novel SAT AllSolutions Solver for Efficient Pre image Computation," in Proceedings of the IEEE International Conference on Design, Automation and Test in Europe, Volume 1, February 2004.

[5] M.Malmgren,"An infrastructure for database tamper detection and forensic analysis and forensic analysis", Honor Thesis university of Arizona, may 2007.

[6] J. Mena, Investigative Data Mining for Security and Criminal Detection, Butterworth Heinemann, 2003.

[7] K. E. Pavlou and R. T. Snodgrass, "Forensic Analysis of Database Tampering," in Proceedings of the ACM SIGMOD International Conference on Management of Data, pp. 109-120, Chicago, June, 2006.

[8] K. E. Pavlou and R. T. Snodgrass, "Forensic Analysis of Database Tampering," ACM Transactions on Database Systems33 (4): Article 30, 47+25 pages, November 2008.

[9] S. Sheng and M. S. Hsiao, "Efficient Pre image Computation Using A Novel Success-Driven ATPG," in Proceedings of the IEEE International Conference on Design, Automation and Test in Europe, Volume 1, March 2003.
[10] R. T. Snodgrass, S. S. Yao, and C. Collberg, "Tamper Detection in Audit Logs," in Proceedings of the International Conference on Very Large Databases, pp. 504-515, Toronto, Canada, September 2004.

[11] C. Strachey, "Bitwise operations," Communications of the ACM4 (3):146, March 1961.

[12] U.S. Dept. of Health \& Human Services, The Health Insurance Portability and Accountability Act (HIPAA), 1996, http://www.cms.hhs.gov/HIPAAGenInfo/ (accessed April 16, 2009).

[13] U.S. Public Law No. 107-204, 116 Stat. 745.The Public Company Accounting Reform and Investor Protection Act, 2002.

[14] K. E. Pavlou and R. T. Snodgrass (2010, April).The Tiled Bitmap Forensic Analysis Algorithm. IEEE Transactions on Knowledge and Data Engineering, 22(4):590-601.

[15] "Forensic Analysis of Database Tampering", K.E. Pavlou and R.T.Snodgrass, Proc. ACM SIGMOD Int'l Conf. Management of Data, pp. 109-120, June 2006.

[16] Amit Basu, Article on Forensic Tamper Detection is SQL Server Tables, http://www.sqlsecurity.com

[17] S. Haber and W. S. Stornetta, "How to Time-Stamp a Digital Document," Journal of Cryptology 3:99-111, 1999.

[18] Mostar, Bosnina and Herzegovina, 2009 International Conference on Computer Engineering and Applications IPCSIT vol.2 (2011) (O) (2011) IACSIT Press, Singapore

[19] Pallavi D Abhonkar et al, / (IJCSIT) International Journal of Computer Science and Information Technologies, Vol. 3 (5), 2012, 5078-5085. 